\title{
Directional selection at gene expression level contributes to the speciation of
}

\section{Asian rice cultivars}

$1 \quad$ Lihong $\mathrm{Xie}^{1 \#}$, Kehan $\mathrm{Yu}^{1 \#}$, Dongjing Chen ${ }^{1 *}$

2 1. AmazingX Academy, Baoli Zhongchen Tower, Foshan 528300, Guangdong,

3 People's Republic of China

$4 \quad$ \# these authors contribute equally to this work.

$5 *$ corresponding author: sysucdj@amazingx.org

6

7 


\section{Abstract}

Differences in expression levels play important roles in phenotypic variation across species, especially those closely related species with limited genomic differences. Therefore, studying gene evolution at expression level is important for illustrating phenotypic differentiation between species, such as the two Asian rice cultivars, Oryza sativa L. ssp. indica and Oryza sativa L. ssp. japonica. In this study, we evaluated the gene expression variation at inter-subspecies and intra-subspecies level using transcriptome data from seedlings of three indica and japonica rice and defined four groups of genes under different natural selections. We found a substantial of genes (about 79\%) that are under stabilizing selection at the expression level in both subspecies, while about $16 \%$ of genes are under directional selection. Genes under directional selection have higher expression level and lower expression variation than those under stabilizing selection, which suggest a potential explanation to subspecies adaptation to different environments and interspecific phenotypic differences. Subsequent functional enrichment analysis of genes under directional selection shows that indica rice have experienced the adaptation to environmental stresses, and also variation, which may guide to rice breeding and yield improvement.

Key words: japonica rice; indica rice; gene expression evolution; speciation, adaptation

\section{Introduction}

Rice grown in Asia (Oryza sativa L.) is a very important food crop for China and the world (Ruan Bosheng, 2008), and more than half of the world's population uses it as a staple food (Sasaki \& Burr, 2000). It is mainly divided into two different subspecies, namely the subspecies Oryza sativa L. ssp. indica (indica) and the subspecies Oryza sativa L. ssp. japonica (japonica). It is speculated that japonica and indica rice were 
domesticated 9,000 years ago (Purugganan \& Fuller, 2009), but debate about their origins still exists. The first model, the single-source model, indicated that the two main subspecies of Asian rice, indica and japonica, were domesticated from wild rice (O. rufipogon.) and then differentiated (Ting, Y., 1957) (Lu et al. al., 2002; Wang et al., 2008). In contrast, the second model, the multiple independent domestication model, proposes that the two main rice types are domesticated separately (Oka \& Morishima, 1982). Indica rice evolved from wild rice. Because, japonica rice is the continuous evolution and artificial selection of indica rice in the process of people's continuous introduction to high latitudes and high altitude areas (Lu Baorong et al., 2009). Therefore, indica rice is the basic type, and japonica rice is the variant type (Molina et al., 2011). The latter has been supported by many researchers after the observation of the strong genetic differentiation between indica and japonica and the development of several systems of rice domestication (Wang, X. et al., 1984). The third origin model claims that indica and japonica were independently domesticated or at least domesticated twice and then differentiated from wild rice (O. rufipogon.) (Garris et al., 2005; Second, 1982).

In most cases, indica rice ecotypes are mainly distributed in low-latitude or high-altitude tropical and subtropical rice growing areas, while japonica rice ecotypes are mainly distributed in high-latitude temperate areas (Lu et al., 2009). Due to long-term adaptation to different ecological environments, japonica rice and indica rice have differences in morphological characteristics, agronomic traits, and genes. In terms of morphological characteristics, the stems of indica rice are thicker, and the plant height is generally more than 1 meter. The tillering ability is stronger, the leaf color is lighter, the grains are slender, easy to fall, and the rice yield rate is low. However, japonica rice generally has a thin stem and a plant height of $75-95 \mathrm{~cm}$. Traditional japonica rice varieties have a lower tillering ability than indica rice. The leaves are darker, the grains are short and round, and they are not easy to shatter, and the rice yield rate is higher. (Flower Encyclopedia; Xu Zhengjin et al., 2003). In terms of agronomic characteristics, indica rice with a short growth period is more resistant 
to humidity, heat, and strong light, but not cold tolerant. After being hulled into indica rice, the transparency of the rice grains is low. Because it contains about $20 \%$ amylose, indica rice is drier and looser when cooked. However, japonica rice, which has a long growth period, generally only matures once a year, is more cold-tolerant and tolerant to low light, but not tolerant to high temperatures. After being hulled into japonica rice, the rice grains have high transparency(Douding.com). Because it contains less amylose, less than 15\%, it is medium-viscosity (Report on Rice Factory Seedling Breeding-Douding.com), and its cooked food characteristics are between glutinous rice and indica rice (Jiang Jian et al., 2001; Miao Xiangwei \& Wang Dexin, 2009; Xu Hai et al., 2007).

We want to study how the advantages of gene expression evolution between japonica rice and indica rice are reflected in the phenotype. Japonica rice and indica rice are two subspecies with similar evolutionary distances and similar genomes. Because the differences between species caused by DNA sequences are relatively small, the differences caused by gene expression regulation are very important for the interpretation of phenotypic differences.

Previous studies on phenotypic differences in species focused on the evolution of gene lineage to study species differentiation (Onishi et al., 2007; Ting et al., 2000), but a small number of study focused on the study of transcriptomes. The transcriptome is a collection of all transcription products in a cell under a specific developmental stage or physiological condition of a species. Understanding the transcriptome is of great significance for explaining the functional elements of the genome and the phenotypic traits controlled by its line (Wang et al., 2009). Therefore, we want to study the influence of the transcriptome on the differences in gene expression and the changes in traits. This will provide great research significance for species differentiation.

In order to measure the extent and specific traits of the differences between Asian 
98 cultivated rice subspecies caused by gene expression levels, we divided genes into 99 four categories according to the differences in gene expression between subspecies and within subspecies: (1) genes subjected to directional selection: genes with large differences in expression between species and small differences in expression within species are the genes that continue to strengthen the selection pressure for a certain trait among populations. These genes are also the main genes that cause differences in traits between species (Mitchell-Olds et al., 2007; Rieseberg et al., 2002). (2) Genes subjected to stabilizing selection: genes with small differences between species and within species are the continuous selection pressure for interspecies traits in the population. This natural selection will not lead to population differentiation (Charlesworth et al., 1982). (3) Genetic drift: genes with large differences between species and within species are a type of gene affected by random factors in the population (Melo \& Marroig, 2015; Nielsen, 2005). (4) Complex scenario: genes with small inter-species differences and large intra-species differences. After the identification of natural selection, we analyze for gene enrichment of the genes subject to directional selection and understand its specific traits and functions (Subramanian et al., 2005).

This article studies the following scientific issues. First, whether the genes subject to directional selection in expression are the genes that cause the phenotypic differences between japonica rice and indica rice. Second, whether the functions of these genes are related to phenotypic differences.

The purpose and significance of this research are mainly to increase yield and breeding. It is to study that this species is closely related to the food production of all human beings, and to analyze the reasons for the differences in rice phenotypes so that people can better control the traits to achieve the ideal yield and quality of rice. 
The acquisition of rice transcriptome data and the measurement of gene expression levels

We obtained the seedling transcriptome data of three different indica and japonica rice lines (Indica: Khao Dawk Mali 105, Guangluai 4, and Rathuwee; Japonica: Taipei 309, Heukgyeong, and Nipponbare) from Wen et al. (Wen et al., 2016). Subsequently, we used hisat2 (version: 2.2.0, default parameters) to map the read length of the original sequencing data of all samples back to the japonica rice reference genome (ensembl, IRGSP-1.0), and used stringtie (version: 2.1.6; parameters: -e, Other defaults) to calculate the TPM (transcript per million) of the protein-coding gene (ensembl, IRGSP-1.0.51). TPM was used as the expression level of the protein-coding gene for subsequent analysis.

\section{Principal component analysis}

After obtaining gene expression levels, we use principal components to analyze the differences between samples. First, the gene-specific TPM uses the R method vegdist to calculate the dissimilarity index between samples, then uses the R method prcomp to calculate the principal component of the sample for the index, and finally uses the $\mathrm{R}$ method ggbiplot to visualize the data. (Figure 1, grouping, principle

\section{Identification of genes under four types of natural selection}

We adopted the method of Yeh et al. According to whether there are significant differences in gene expression between species and within species, we divided them into four categories: directional selection: significant differences between species and insignificant differences within species; stabilizing selection: both difference between interspecies and difference between intraspecies are not significant; genetic drift: interspecies and intraspecies differences are both significant; complex scenario: 
158 interspecies differences are not significant, and intraspecies differences are significant

159 (Yeh et al., 2014). We use a linear model to measure whether there are significant

160 differences in gene expression between species and within species.

$161 \quad$ For interspecies:

$$
y_{i}=\mu+S_{i}+\varepsilon_{i}
$$

Among them, $y_{i}$ represents the expression level of the protein coding gene of species i, $\mu$ is the basic expression level, $S_{i}$ is the effect of species i, and $\varepsilon_{i}$ is the residual. If the $\mathrm{P}$ value of the linear model is less than 0.05 , it is defined as significant difference between species.

For intraspecies:

$$
y_{j}=\mu+L_{j}+\varepsilon_{j}
$$

Among them, $y_{j}$ represents the expression level of the protein coding gene of line $\mathrm{j}, \mu$ is the basic expression level, $L_{j}$ is the effect of line $\mathrm{j}, \varepsilon_{j}$ and is the residual. If the $\mathrm{P}$ value of the linear model is less than 0.05 , it is defined as significant intraspecies difference.

\section{Functional enrichment analysis}

In order to find genes and functions related to subspecies differentiation, we used RiceNETDB (http://bis.zju.edu.cn/ricenetdb/) to carry out Gene Ontology (Gene Ontology) on indica and japonica species-specific directional selection genes., GO) enrichment analysis. The three functional branches of GO, the biological process, molecular function and cellular component, are all used for functional enrichment analysis. 


\section{Results}

\section{Processing and expression analysis of Asian cultivated rice transcriptome data}

We obtained the seedling transcriptome data of three indica rice lines (Khao Dawk Mali 105, Guangluai 4 and Rathuwee) and three japonica rice lines (Taipei 309, Heukgyeong and Nipponbare) from the research of Wen et al. (Wen et al., 2016)(Table 1). After hisat2 (version 2.2.0), the initial read length was posted to the reference genome (ensembl, IRGSP-1.0). Among the samples with a total read length of $18,818,192$ to $20,326,850$, it was found that the only response rate of the transcriptome sequencing read length was between 63.41 and $68.40 \%$. For the 35,775 protein-coding genes that have been annotated, the number of genes detected to be expressed (TPM greater than 0) ranged from 28,021 to 29,092 (78.33 to $81.32 \%$ ); while higher expression levels were detected (TPM greater than or equal to 5), the number of genes is between $16,942-17,807(47.36-49.77 \%)$. It shows that in our subsequent analysis, about $50 \%$ of the genes can be judged to be credible and selected because of their higher expression levels.

Table 1. Indica and Japonica transcriptome statistics

\begin{tabular}{|c|c|c|c|c|c|c|}
\hline Subspecies & SRR id & $\begin{array}{l}\text { Accession } \\
\text { name }\end{array}$ & Total reads & $\begin{array}{l}\text { Uniquely } \\
\text { mapped }\end{array}$ & $\begin{array}{l}\# \text { of } \\
\text { detected } \\
\text { PCGs } \\
(\mathrm{TPM}>0) \\
\end{array}$ & $\begin{array}{l}\# \quad \text { of } \\
\text { detected } \\
\text { PCGs } \\
(\mathrm{TPM} \geq 5)\end{array}$ \\
\hline \multirow[t]{3}{*}{ indica } & SRR2154082 & Guangluai4 & $20,326,850$ & $\begin{array}{l}13,336,411 \\
(65.61 \%)\end{array}$ & $\begin{array}{l}28,021 \\
(78.33 \%)\end{array}$ & $\begin{array}{l}16,942 \\
(47.36 \%)\end{array}$ \\
\hline & SRR2154083 & KDM105 & $20,216,686$ & $\begin{array}{l}12,820,328 \\
(63.41 \%)\end{array}$ & $\begin{array}{l}28,316 \\
(79.15 \%)\end{array}$ & $\begin{array}{l}17,367 \\
(48.55 \%)\end{array}$ \\
\hline & SRR2154084 & Rathuwee & $19,876,912$ & $\begin{array}{l}12,716,807 \\
(63.98 \%)\end{array}$ & $\begin{array}{l}28,240 \\
(78.94 \%)\end{array}$ & $\begin{array}{l}17,428 \\
(48.72 \%)\end{array}$ \\
\hline \multirow[t]{3}{*}{ japonica } & SRR2154085 & Heukgyeong & $19,889,198$ & $\begin{array}{l}13,584,731 \\
(68.30 \%)\end{array}$ & $\begin{array}{l}28,766 \\
(80.41 \%)\end{array}$ & $\begin{array}{l}17,312 \\
(48.39 \%)\end{array}$ \\
\hline & SRR2154086 & Nipponbare & $18,818,192$ & $\begin{array}{l}12,871,620 \\
(68.40 \%)\end{array}$ & $\begin{array}{l}29,300 \\
(81.90 \%)\end{array}$ & $\begin{array}{l}17,807 \\
(49.77 \%)\end{array}$ \\
\hline & SRR2154087 & Taipei309 & $19,094,551$ & $\begin{array}{l}13,013,267 \\
(68.15 \%)\end{array}$ & $\begin{array}{l}29,092 \\
(81.32 \%)\end{array}$ & $\begin{array}{l}17,475 \\
(48.85 \%)\end{array}$ \\
\hline
\end{tabular}


204 Subsequently, we conducted a principal component analysis of six lines from two

205 cultivated rice subspecies, and found that the difference between the samples of indica and japonica is relatively large (the first principal component PC1 can explain $64.2 \%$ of the variation between the samples), while the subspecies differences in the internal samples are small (the second principal component PC2 only explains $12.5 \%$ of the variation between samples ) (Figure 1). It shows that the data is sufficient to define the evolution of expression based on inter-species and intra-species differences (see Materials and Methods).

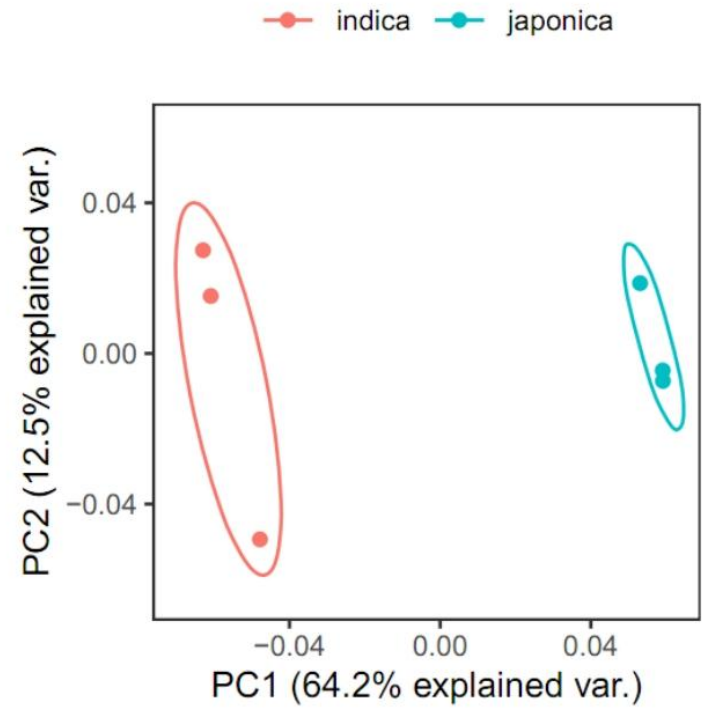

Figure 1. Principal component analysis of the transcriptome of indica and japonica rice samples.

The expression of most genes in indica and japonica rice is affected by stabilizing selection

Indica rice and japonica rice are subspecies of cultivated rice in Asia. They diverged about 0.55 million years ago (Stein et al. 2018, nature genetics). There is only $1 / 6$ difference in genome, and most of them are located on transposons (Campbell et al., 2020; Gao et al., 2015; Ma \& Bennetzen, 2004). Therefore, in addition to the genomic differences between indica and japonica rice, differences in 
gene expression levels are also an important factor explaining the differences in traits between this subspecies (Rieseberg et al., 2002). In order to find out which genes cause differences between subspecies, we divided genes into four categories based on differences in gene expression between and within subspecies: genes subject to directional selection, genes subject to stabilizing selection gene, genetic drift, and complex scenario. Our results show that among the 35,775 genes in indica and japonica, most of the genes are subject to stabilizing selection (indica: 28,501, accounting for 79.67\%; japonica: 28,198 , accounting for $78.82 \%$ ), which is similar to the two subspecies. The genomic differences are relatively small and consistent (Figure 2A,B). For example, the expression difference of the gene Os12g0274700 between and within the two subspecies is relatively small and not significant (Figure 2C, D ). However, there are still quite a few genes subject to targeted selection, $16.25 \%(5,910 / 35,775)$ in indica rice and $16.36 \%(5,854 / 35,775)$ in japonica rice (Figure 1A, B ). For example, in indica rice, the gene Os08g0435900, which is subject to targeted selection, has a large difference in expression between species, while the difference between indica species is not significant (Figure 2C). This gene is chlorophyll ab binding protein P4 (chlorophyll ab binding protein P4), which is located in the chloroplast, and is highly expressed in flag leaves before flowering (Wang et al., 2015) . The gene Os07g0147500, which is subject to directional selection in japonica rice, has a larger expression difference between japonica rice and indica rice, but the expression difference in japonica rice is not significant (Figure 2D). This gene is a $10 \mathrm{kDa}$ polypeptide located in the photosystem II of the chloroplast, and is highly expressed in flower buds, leaves after anthesis, and grain-filled seeds (Wang et al., 2015) . In addition, genetic drift genes whose expression is affected by random factors, that is, genes that are not significantly different between species and within species, account for about $3.09 \sim 3.94 \%$ of the two subspecies ; the expression of very few genes differs within species Significant but not significant differences between species, it is a complex situation, accounting for 0.72 to $0.88 \%$ (Figure $2 \mathrm{~A}, \mathrm{~B}$ ). 
Indica

A

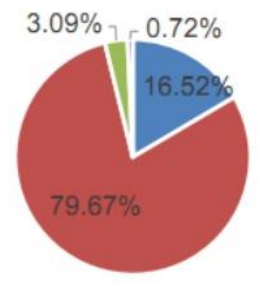

Japonica

B

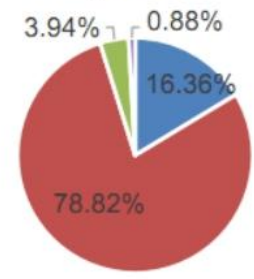

- directional

- stablizing

" genetic drift

- complex scenario

C Intraspecific variation in Indica

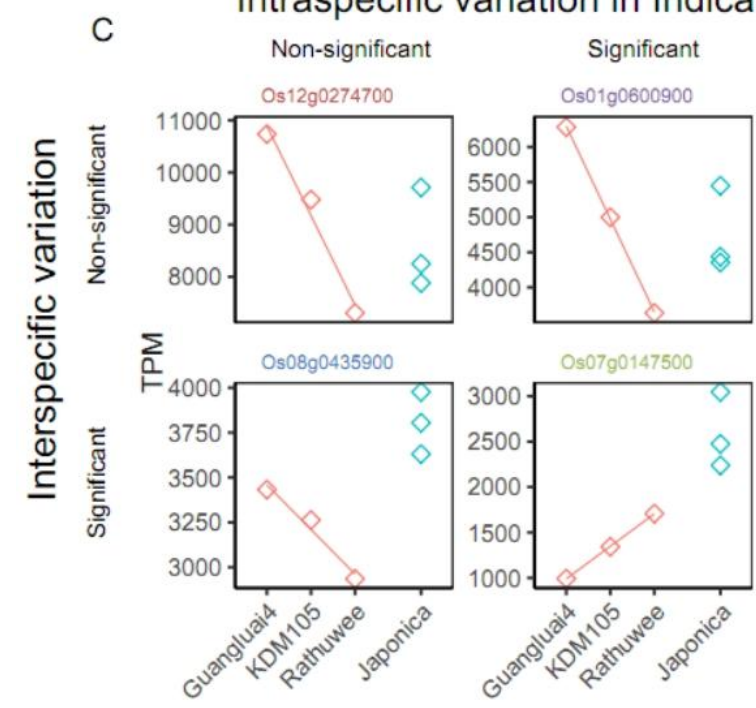

Intraspecific variation in Japonica

Non-significant Significant
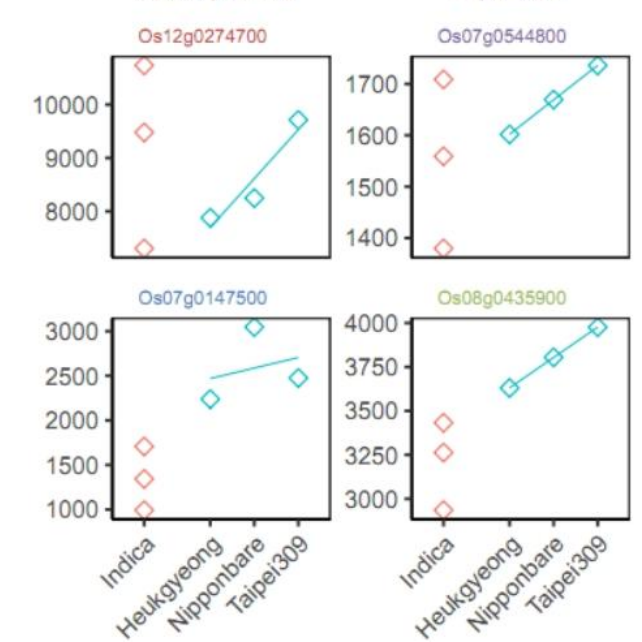

Figure 2. Evolutionary pattern of gene expression in indica and japonica rice. The ratio of the four types of expression selection in indica (A) and japonica (B). Graphical representation of the expression of four different selection types of genes in indica (C) and japonica (D). The inter-species and intra-species significance were obtained by one-way analysis of variance (one-way ANOVA). Directional selection: genes with significant differences between species and insignificant differences within species; stabilizing selection: genes with insignificant differences between species and within species; genetic drift: between species Genes with significant differences and intraspecies differences, and complex scenarios: Genes with insignificant differences between species and significant differences within species. Red: Indica rice strain; Blue: Japonica rice strain.

The difference in expression level of directional selection genes and stabilizing 
270 Species differentiation is affected by two types of genes: stabilizing selection and

271 directional selection. Genes with small differences between species and within species

272 when genes are subjected to stabilizing selection are the continuous selection pressure

273 for interspecific traits in the population, and this natural selection will not lead to

274 population differentiation (Charlesworth et al., 1982); genes subjected to directional

275 selection are genes with large differences in expression between species and small

276 differences in expression within species. They are the continuous strengthening of

277 selective pressure for a trait among populations, and are the main genes that cause

278 differences in traits between species (Mitchell-Olds et al., 2007; Rieseberg et al.,

279 2002). Through previous research and analysis, we found that indica rice and

280 japonica rice are mainly subject to two types of genes, namely stabilizing selection

281 and directional selection, respectively. We analyze the differences in their expression

282 levels between subspecies strains. We get TPM median of indica gene level is 7.3773,

283 TPM median of gene subjected to stabilizing selection is 3.6448 . The expression

284 level of directional selection genes in indica rice was significantly greater than that of

285 stabilizing selection genes (Figure 3A Wilkerson rank sum test, $\mathrm{p}<0.001$ ). In addition,

286 the coefficient of variation (0.1364) of directional selection genes was significantly

287 lower than that of stabilizing selection genes (0.2451) (Figure 3A Wilkerson rank sum

288 test, $\mathrm{p}<0.001)$. We also found the same situation in japonica

289 rice. The median TPM expression level of the directional selection gene in japonica

290 rice was 9.4966, and the gene expression level of the stabilizing selection gene

291 was 3.4431. The expression level of directional selection genes was significantly

292 greater than that of stabilizing selection genes (Figure 3B Wilkerson rank sum

293 test, $\mathrm{p}<0.001$ ). Similarly, the coefficient of variation of japonica gene subjected to

294 directional selection (0.1106) is significantly lower than the coefficient of variation of

295 gene subjected to stabilizing selection (0.1904) (Figure 3B Weierkesen rank sum

296 test, $\mathrm{P}<$ from 0.001). Therefore, from the data of gene expression level and variation

297 level, we can conclude that the expression level of targeted selection genes is higher

298 and the variation level is lower. Directional selection genes play a more important role

299 in the process of subspecies differentiation than stabilizing selection genes. 
A

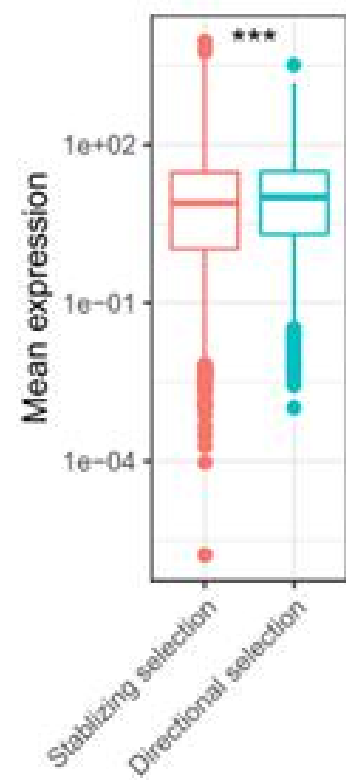

indica

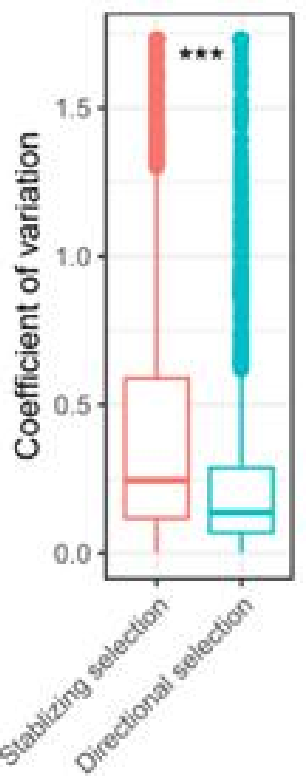

B

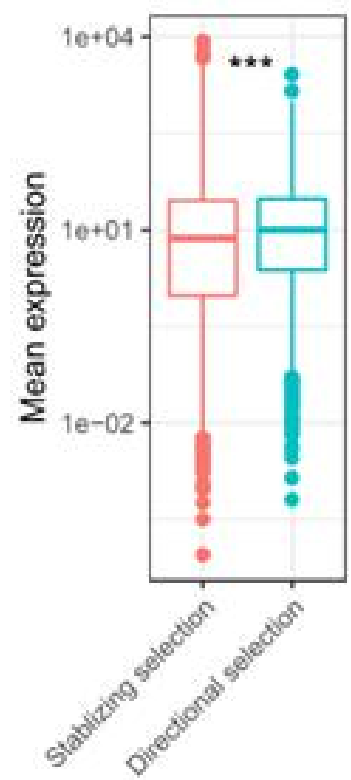

japonica

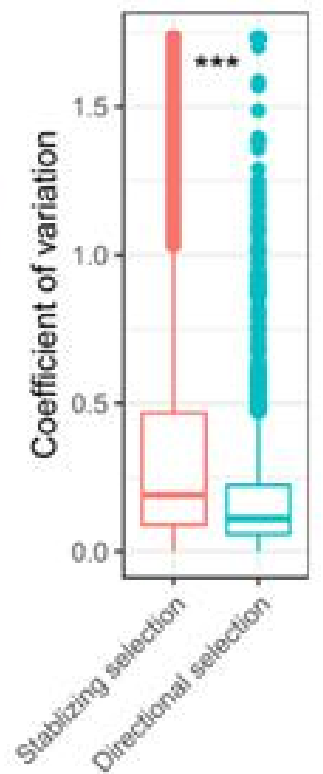

Figure 3. The expression and variation levels of directional selection and stabilizing selection in indica (A) and japonica (B). *** Wilcoxon rank sum test, $\mathrm{p}<0.001$. Red: stabilizing selection gene; green: directional selection gene.

\section{Indica rice species-specific targeted selection genes will enrich biological pathways to cope with environmental pressures}

In order to measure the phenotypic difference between indica and japonica in gene expression levels, we focused on species-specific directional selection of genes. Among the 5,910 indica-directional selection genes identified, 298 were indica-specific; among the 5,854 japonica-directional selection genes, 242 were japonica-specific. Subsequently, we will conduct gene function enrichment analysis for these subspecies-specific targeted selections (see Materials and Methods) to obtain functional pathways related to phenotypic differences. In addition to enriching a large number of common functional pathways, we found that indica rice-specific targeted genes also specifically enriched two types of biological pathways (Figure 4). The first category is related to coping with environmental stress, such as the response to stimulus, response to stress, response to abiotic stimulus (Figure 4A). This is related 
319 to the different geographical distribution, temperature and light adaptation of the two

320 subspecies of indica and japonica (Foll \& Gaggiotti, 2006; Mahgoub, 2019; Wu et al.,

321 2019). The second category is the biosynthesis and metabolism, especially the

322 Biopolymer Metabolism, Carbohydrate Metabolism, Protein biosynthesis,

323 biosynthesis Macromolecule, etc (Figure 4A). This type of pathway may be related to

324 the difference in starch and protein composition between indica and japonica rice. For

325 example, indica rice has a higher amylose content than japonica rice (DuPont \&

326 Altenbach, 2003; Padilla-González et al., 2019). In addition, indica

327 subspecies-specific targeted selection genes also have characteristics in the location of

328 cellular components, for example, they are specifically located

329 in non-membrane-bound organelles (intracellular non-membrane-bound organelle,

330 external encapsulating structure). The molecule function also exist specificity, such as

331 the activity of the specific structure of the molecules enriched (Structural Molecular

332 Activity), transferase activity, etc. 

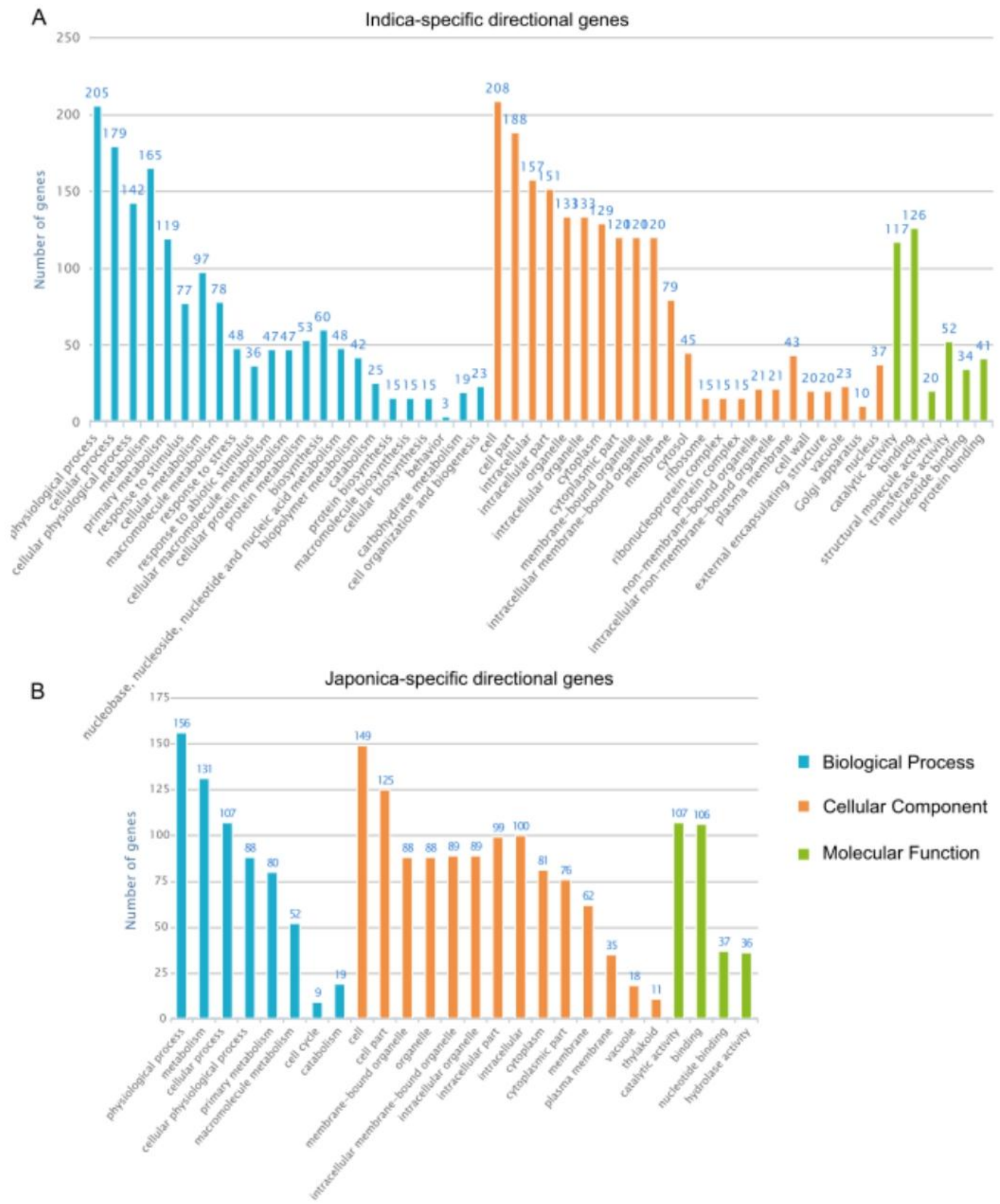

334 Figure 4. Gene Ontology (GO) functional enrichment analysis of species-specific directional selection genes in indica (A) and japonica (B). Blue: biological processes; orange: cellular components; green: molecular functions.

\section{Discussion}

In the process of natural differentiation or artificial selection of species, the core gene regions affected by selection pressure are of concern to a wide range of researchers. If gene flow continues to affect species undergoing natural differentiation or artificial 
selection, under different selection pressures, different regions of the same genome will express different evolutionary results ( $\mathrm{He}$ et al., 2011), such as gene introgression. Differences in genomic DNA and changes in epigenetics are the two main factors that cause differences between species. Unlike in the past, which mainly focused on the change of DNA sequence to study species differentiation (He et al., 2011), in recent years, more studies have focused on the transcriptome level and the expression level to explore the factors of species evolution. These studies have found that gene expression has a wide range of adaptability and is related to species differentiation (Guo et al., 2016; Wen et al., 2016; Yeh et al., 2014). In the past, some scholars studied the effects of changes in the species transcriptome on the evolution of gene expression, and gene chips. But our research has data advantages. We study the evolution of gene expression, which makes it easier to accurately find genes that differentiate between species than pure differential expression. In addition, we use more types of strains and effective methods to obtain more accurate transcriptomes (Ding et al., 2007; Goff et al., 2002; Huang et al., 2012; Wang et al., $2014)$.

If the effects on rice differentiation are studied by observing changes in genome, this will have limitations and deficiencies, such as only studying wild rice and neglecting artificial selection (Guo et al., 2016) , or lack of measuring the internal expression variation of multiple strains between subspecies (Guo et al., 2016), or lack of sequencing data of tissue or developmental stages at that time (Wen et al., 2016). We use the linear model method to study the interspecific and intraspecific expression differences of the seedling transcriptome data of six lines of japonica rice and indica rice, and divide the genes into four selected gene types. We found that most of the subspecies with a higher degree of genome similarity are genes that are subjected to stabilizing selection, followed by genes that are subject to targeted selection. Later, in further comparing the expression levels and expression variation levels of genes subject to stabilizing selection and targeted selection, we found that targeted selection genes have higher expression levels and lower variation levels than stabilizing 
selection genes. This illustrates the importance of directional selection of genes in species differentiation. Finally, in the enrichment analysis of genes subject to targeted selection, we found that these genes are related to environmental pressure. This can indicate that the directional selection genes of japonica rice may have been affected by environmental pressure, which led to the differentiation of indica rice. The study of species evolution from the perspective of the transcriptome can provide different perspectives and depths.

Our research has two main shortcomings. First, from the data level, we only collected the transcriptome data of indica and japonica rice seedlings but missed the transcriptome data of other tissues or developmental stages. Future research can use more extensive and comprehensive organization and developmental stages to carry out more in-depth research. Second, there are many models for the evolution of cultivated rice. There are single-source models, that is they are domesticated together and then differentiated. There are also multiple independent domestication models, that is indica rice originates from japonica rice, and there are independent-origin models, that is completely independent domestication. In addition, wild rice, the ancestor of cultivated rice, is also controversial (He et al., 2011; Stein et al., 2018). However, we only studied the transcriptome of two cultivated rice. Future research can focus on the transcriptome of different wild rice. Although our results are insufficient, we have discovered the contribution of the transcriptome of cultivated rice seedlings to species differentiation. This lays the foundation for future research on the impact of changes in gene expression levels on species differentiation and gives certain prospective results, especially the impact of artificial selection on cultivated rice.

\section{Funding}

This research received no external funding.

\section{Conflict of interest}

The authors declare no competing interests. 


\section{References}

Sasaki T, Burr B. International Rice Genome Sequencing Project: The effort to completely sequence the rice genome. Curr Opin Plant Biol . 2000;3:138-142.

Ting, Y. (1957). The origin and evolution of cultivated rice in China. Acta Agron.

Campbell, MT, Du, Q., Liu, K., Sharma, S., Zhang, C., \& Walia, H. (2020). Characterization of the transcriptional divergence between the subspecies of cultivated rice (Oryza sativa). BMC genomics , 21 , 1-16.

Charlesworth, B., Lande, R., \& Slatkin, M. (1982). A NEO-DARWINIAN COMMENTARY ON MACROEVOLUTION. Evolution , 36 (3), 474-498. https://doi.org/10.1111/j. 1558-5646.1982.tb05068.x

DuPont, FM, \& Altenbach, SB (2003). Molecular and biochemical impacts of environmental factors on wheat grain development and protein synthesis. Journal of cereal science, 38 (2), 133-146.

Foll, M., \& Gaggiotti, O. (2006). Identifying the environmental factors that determine the genetic structure of populations. Genetics, 174 875-891. https://doi.org/10.1534/genetics.106.059451

Gao, D., Jiang, N., Wing, RA, Jiang, J., \& Jackson, SA (2015). Transposons play an important role in the evolution and diversification of centromeres among closely related species [Original Research]. Frontiers in Plant Science , 6 (216). https://doi.org/10.3389/fpls.2015.00216

Garris, AJ, Tai, TH, Coburn, J., Kresovich, S., \& McCouch, S. (2005). Genetic structure and diversity in Oryza sativa L. Genetics, 169 (3), 1631-1638. 
Goff, SA, Ricke, D., Lan, TH, Presting, G., Wang, R., Dunn, M., Glazebrook, J., Sessions, A., Oeller, P., Varma, H., Hadley, D., Hutchison, D., Martin, C., Katagiri, F., Lange, BM, Moughamer, T., Xia, Y., Budworth, P., Zhong, J., Miguel, T., Paszkowski, U ., Zhang, S., Colbert, M., Sun, WL, Chen, L., Cooper, B., Park, S., Wood, TC, Mao, L., Quail, P., Wing, R., Dean, R., Yu, Y., Zharkikh, A., Shen, R., Sahasrabudhe, S., Thomas, A., Cannings, R., Gutin, A., Pruss, D., Reid, J., Tavtigian, S., Mitchell, J., Eldredge, G., Scholl, T., Miller, RM, Bhatnagar, S., Adey, N., Rubano, T., Tusneem, N., Robinson, R., Feldhaus , J., Macalma, T., Oliphant, A., \& Briggs, S. (2002). A draft sequence of the rice genome (Oryza sativa L. ssp. japonica). Science $\quad 296$ (5565), 92-100 . https://doi.org/10.1126/science.1068275

Guo, J., Liu, R., Huang, L., Zheng, X.-M., Liu, P.-L., Du, Y.-S., Cai, Z., Zhou, L., Wei , X.-H., \& Zhang, F.-M. (2016). Widespread and adaptive alterations in genome-wide gene expression associated with ecological divergence of two Oryza species. Molecular biology and evolution, 33 (1), 62- 78.

He, Z., Zhai, W., Wen, H., Tang, T., Wang, Y., Lu, X., Greenberg, AJ, Hudson, RR, Wu, C.-I., \& Shi, S . (2011). Two evolutionary histories in the genome of rice: the roles of domestication genes. PLoS genetics , 7 (6), e1002100.

Huang, X., Kurata, N., Wei, X., Wang, ZX, Wang, A., Zhao, Q., Zhao, Y., Liu, K., Lu, H., Li, W., Guo , Y., Lu, Y., Zhou, C., Fan, D., Weng, Q., Zhu, C., Huang, T., Zhang, L., Wang, Y., Feng, L., Furuumi , H., Kubo, T., Miyabayashi, T., Yuan, X., Xu, Q., Dong, G., Zhan, Q., Li, C., Fujiyama, A., Toyoda, A., Lu , T., Feng, Q., Qian, Q., Li, J., \& Han, B. (2012). A map of rice genome variation reveals the origin of cultivated rice. Nature , 490 (7421), 497501. https://doi.org/10.1038/nature11532

Lu, B.-R., Cai, X., \& Xin, J. (2009). Efficient indica and japonica rice identification based on the InDel molecular method: Its implication in rice breeding and evolutionary research. Progress in Natural Science, 19 (10), 1241-1252. https://doi.org/https://doi.org/10.1016/j.pnsc.2009.01.011 
Lu, BR, Zheng, KL, Qian, HR, \& Zhuang, JY (2002). Genetic differentiation of wild relatives of rice as assessed by RFLP analysis. Theor Appl Genet , 106 (1), 101-106. https:// doi.org/10.1007/s00122-002-1013-2

Ma, J., \& Bennetzen, JL (2004). Rapid recent growth and divergence of rice nuclear genomes. Proceedings of the National Academy of Sciences of the United States of America , 101 (34), 12404-12410. doi.org/10.1073/pnas.0403715101

Mahgoub, AMMA (2019). The impact of five environmental factors on species distribution and weed community structure in the coastal farmland and adjacent territories in the northwest delta region, Egypt. Heliyon , 5 (4), e01441. https://doi. org/https://doi.org/10.1016/j.heliyon.2019.e01441

Melo, D., \& Marroig, G. (2015). Directional selection can drive the evolution of modularity in complex traits. Proceedings of the National Academy of Sciences , 112 (2), 470-475. https://doi.org/10.1073/pnas.1322632112

Mitchell-Olds, T., Willis, JH, \& Goldstein, DB (2007). Which evolutionary processes influence natural genetic variation for phenotypic traits? Nature Reviews Genetics , 8 (11), 845-856. https://doi.org/10.1038/nrg2207

Molina, J., Sikora, M., Garud, N., Flowers, JM, Rubinstein, S., Reynolds, A., Huang, P., Jackson, S., Schaal, BA, \& Bustamante, CD (2011). Molecular evidence for a single evolutionary origin of domesticated rice. Proceedings of the National Academy of Sciences , 108 (20), 8351-8356.

Nielsen, R. (2005). Molecular signatures of natural selection. Annu Rev Genet, 39 , 197-218. https://doi.org/10.1146/annurev.genet.39.073003.112420

Oka, H.-I., \& Morishima, H. (1982). Phylogenetic differentiation of cultivated rice, XXIII. Potentiality of wild progenitors to evolve the indica and japonica types of rice cultivars. Euphytica, 31 (1), 41-50 .

Onishi, K., Takagi, K., Kontani, M., Tanaka, T., \& Sano, Y. (2007). Different patterns of genealogical relationships found in the two major QTLs causing reduction of seed shattering during rice domestication. Genome , 50 (8), 757-766. https://doi.org/10.1139/g07-051 
Padilla-González, GF, Frey, M., Gómez-Zeledón, J., Da Costa, FB, \& Spring, O. (2019). Metabolomic and gene expression approaches reveal the developmental and environmental regulation of the secondary metabolism of yacón ( Smallanthus sonchifolius, Asteraceae). Scientific Reports , 9 (1), 13178. https://doi.org/10.1038/s41598-019-49246-2

Purugganan, MD, \& Fuller, DQ (2009). The nature of selection during plant domestication.

Nature

457

(7231), 843-848. https://doi.org/10.1038/nature07895

Rieseberg, LH, Widmer, A., Arntz, AM, \& Burke, JM (2002). Directional selection is the primary cause of phenotypic diversification. Proceedings of the National Academy of Sciences of the United States of America , 99 (19), 12242-12245. https://doi.org/10.1073/pnas.192360899

Second, G. (1982). Origin of the genic diversity of cultivated rice (Oryza spp.): study of the polymorphism scored at 40 isozyme loci. The Japanese journal of genetics , 57 (1), 25-57.

Stein, JC, Yu, Y., Copetti, D., Zwickl, DJ, Zhang, L., Zhang, C., Chougule, K., Gao, D., Iwata, A., Goicoechea, JL, Wei, S ., Wang, J., Liao, Y., Wang, M., Jacquemin, J., Becker, C., Kudrna, D., Zhang, J., Londono, CEM, Song, X., Lee, S. , Sanchez, P., Zuccolo, A., Ammiraju, JSS, Talag, J., Danowitz, A., Rivera, LF, Gschwend, AR, Noutsos, C., Wu, C.-c., Kao, S. -m., Zeng, J.-w., Wei, F.-j., Zhao, Q., Feng, Q., El Baidouri, M., Carpentier, M.-C., Lasserre, E., Cooke , R., Rosa Farias, D. d., da Maia, LC, dos Santos, RS, Nyberg, KG, McNally, KL, Mauleon, R., Alexandrov, N., Schmutz, J., Flowers, D., Fan, C., Weigel, D., Jena, KK, Wicker, T., Chen, M., Han, B., Henry, R., Hsing, Y.-i. C., Kurata, N., de Oliveira, AC, Panaud, O., Jackson, SA, Machado, CA, Sanderson, MJ, Long, M., Ware, D., \& Wing, RA (2018). Genomes of 13 domesticated and wild rice relatives highlight genetic conservation, turnover and innovation across the genus Oryza. Nature Genetics , 50 (2), 285-296. https://doi.org/10.103 8/s41588-018-0040-0 
Subramanian, A., Tamayo, P., Mootha, VK, Mukherjee, S., Ebert, BL, Gillette, MA, Paulovich, A., Pomeroy, SL, Golub, TR, Lander, ES, \& Mesirov, JP (2005 ). Gene set enrichment analysis: a knowledge-based approach for interpreting genome-wide expression profiles. Proceedings of the National Academy of Sciences of the United States of America, 102 15545-15550. https://doi.org/10.1073/pnas.0506580102

Ting, C.-T., Tsaur, S.-C., \& Wu, C.-I. (2000). The phylogeny of closely related species as revealed by the genealogy of a speciation gene, Odysseus. Proceedings of the National Academy of Sciences , 97 (10), 5313-5316.

Wang, H., Niu, QW, Wu, HW, Liu, J., Ye, J., Yu, N., \& Chua, NH (2015). Analysis of non-coding transcriptome in rice and maize uncovers roles of conserved lncRNAs associated with agriculture traits. Plant $J, 84$ (2), 404-416. https://doi.org/10.1111/tpj.13018

Wang, MX, Zhang, HL, Zhang, DL, Qi, YW, Fan, ZL, Li, DY, Pan, DJ, Cao, YS, Qiu, ZE, Yu, P., Yang, QW, Wang, XK, \& Li, ZC (2008). Genetic structure of Oryza rufipogon Griff. in China. Heredity (Edinb) , 101 (6), 527-535. https://doi.org/10.1038/hdy.2008.61

Wang, X., Kudrna, DA, Pan, Y., Wang, H., Liu, L., Lin, H., Zhang, J., Song, X., Goicoechea, JL, Wing, RA, Zhang, Q ., \& Luo, M. (2014). Global genomic diversity of Oryza sativa varieties revealed by comparative physical mapping. 937-949. https://doi.org/10.1534/genetics.113.159970

Wang, Z., Gerstein, M., \& Snyder, M. (2009). RNA-Seq: a revolutionary tool for transcriptomics. Nat Rev Genet , 10 (1), 57-63. https://doi.org/ $\underline{10.1038 / \operatorname{nrg} 2484}$

Wen, M., Xie, M., He, L., Wang, Y., Shi, S., \& Tang, T. (2016). Expression variations of miRNAs and mRNAs in rice (Oryza sativa). Genome biology and evolution , 8 (11), 3529-3544. 
Wu, Z., Xu, X., Zhang, J., Wiegleb, G., \& Hou, H. (2019). Influence of environmental factors on the genetic variation of the aquatic macrophyte Ranunculus subrigidus on the Qinghai-Tibetan Plateau. BMC Evolutionary Biology, 19 (1), 228. https://doi.org/10.1186/s12862-019-1559-0

Yeh, S.-D., von Grotthuss, M., Gandasetiawan, KA, Jayasekera, S., Xia, X.-Q., Chan, C., Jayaswal, V., \& Ranz, JM (2014). Functional divergence of the miRNA transcriptome at the onset of Drosophila metamorphosis. Molecular biology and evolution, 31 (10), 2557-2572.

Douding Network . https://www.docin.com/p-2595022643.html

Flower Encyclopedia . https://nong.huabaike.com/n/5849.html

Jiang Jian, Li Jinquan, Xu Zhengjin, Zhang Longbu, \& Jin Chenghai . (2001). Differences in the main morphological and anatomical characteristics of indica and japonica rice and their relationship with economic traits . Jilin Agricultural Sciences , 26 (6), 11-15.

Lu Baorong, Cai Xingxing , \& Jin Xin . (2009). High-efficiency molecular identification methods for indica and japonica rice and their significance in rice breeding and evolution. Advances in Natural Science, 019 (006), 628-638.

Miao Xiangwei , \& Wang Dexin . (2009). Research on the correlation between rice subspecies characteristics and economic traits . Seed World (12), 28-31.

Ruan Bosheng . (2008). Creation of a mutant bank of japonica rice " Nipponbare" and genetic analysis of some important traits Zhejiang University ].

Rice Seedling Nursery report - Douding · Https://www.docin.com/p-1870714240.html

Xu , Liu Hongguang, Zhuchun Jie , Yang Li , GUO Yan-hua, Wang Jiayu , Yang Gan Hua , Xu into , Zhengjia Kui , \& Chen Wenfu . (2007). Effects of ecological condition of Subspecies Characteristics and Economic Characters of Indica rice hybrids . Zirankexue progress . 
bioRxiv preprint doi: https://doi.org/10.1101/2021.10.31.466501; this version posted November 2, 2021. The copyright holder for this preprint (which was not certified by peer review) is the author/funder, who has granted bioRxiv a license to display the preprint in perpetuity. It is made available under aCC-BY-NC-ND 4.0 International license.

579 Xu Zhengjin, Li Jinquan, Jiang Jian, Jing Yanhui , Zhang Wenzhong, Chen Wenfu ,

580 \& Zhang Longbu . (2003). Subspecies characteristic traits of indica and japonica hybrids and their relationships with economic traits . Acta Agronomica Sinica, 029 (005), 735 -739.

583 